Meta

Journal des traducteurs

Translators' Journal

\title{
La poesía intervencionista de José María Heredia
}

\section{Jenny Pérez Carrasco}

Volume 50, numéro 4, décembre 2005

Pour une traductologie proactive - Actes

For a Proactive Translatology — Proceedings

Por una traductología proactiva - Actas

URI : https://id.erudit.org/iderudit/019927ar

DOI : https://doi.org/10.7202/019927ar

Aller au sommaire du numéro

Éditeur(s)

Les Presses de l'Université de Montréal

ISSN

0026-0452 (imprimé)

1492-1421 (numérique)

Découvrir la revue

Citer cet article

Pérez Carrasco, J. (2005). La poesía intervencionista de José María Heredia. Meta, 50(4). https://doi.org/10.7202/019927ar

\section{Résumé de l'article}

Dans cet article, nous analyserons la traduction du poème « El mérito de las mujeres » de José María Heredia y Heredia, traduction du poème français « Le mérite des femmes » d'Ernest Wilfrid Legouvé. Cette étude s'insère dans un projet de recherche sur la traduction à Cuba au XIX ${ }^{\mathrm{e}}$ siècle. Nous postulons que la traduction de José María Heredia est le résultat d'interventions délibérées lui permettant de développer un volet particulier de son oeuvre littéraire : les poésies interventionnistes. 


\title{
La poesía intervencionista de José María Heredia
}

\author{
JENNY PÉREZ CARRASCO \\ Université de Montréal, Montréal, Canada \\ jenny.perez-carrasco@umontreal.ca
}

\begin{abstract}
RÉSUMÉ
Dans cet article, nous analyserons la traduction du poème «El mérito de las mujeres » de José María Heredia y Heredia, traduction du poème français « Le mérite des femmes » d'Ernest Wilfrid Legouvé. Cette étude s'insère dans un projet de recherche sur la traduction à Cuba au XIX ${ }^{\mathrm{e}}$ siècle. Nous postulons que la traduction de José María Heredia est le résultat d'interventions délibérées lui permettant de développer un volet particulier de son ouvre littéraire : les poésies interventionnistes.
\end{abstract}

\begin{abstract}
In this article we analyze the Spanish translation of the poem "Le mérite des femmes" written by Ernest Wilfrid Legouvé and translated into Spanish by José María Heredia y Heredia as "El mérito de las mujeres". This study is part of a research project on translation in Cuba in the nineteenth century. We argue that Heredia's translation is the result of set deliberate interventions that allowed him to develop another aspect of his literary work: "interventionist poems".
\end{abstract}

\section{MOTS-CLÉS/KEYWORDS}

traduction, Cuba, José María Heredia y Heredia, intervention délibérée, poésies interventionnistes

Este trabajo constituye un estudio de caso dentro de un proyecto de doctorado cuyo objetivo es analizar la evolución de la traducción y el comportamiento de los traductores en Cuba en el siglo XIX. El presente caso se refiere a la traducción del poema "El mérito de las mujeres" de José María Heredia y Heredia, versión española del poema francés "Le mérite des femmes" de Ernest Wilfrid Legouvé $^{-}$. El análisis del comportamiento o de las estrategias utilizadas por los traductores exige, además del imprescindible estudio comparativo de los textos, deternerse en el contexto histórico y social de la traducción, pero también en el contexto personal del traductor.

Planteamos que la traducción de José María Heredia es el resultado de la intervención deliberada del traductor, entendida como apropiación del texto original, alejada de la literalidad del texto de partida a partir de un procedimiento no neutro y no objetivo. Poco se sabe de Heredia traductor, con excepción de trabajos hechos por la investigadora y traductora cubana, Carmen Suárez de León desde hace algunos años, así como por otros investigadores cubanos. Las investigaciones han sido más bien dedicadas a la obra literaria de Heredia. A través de este trabajo comparativo de la traducción de Heredia y del original, queremos mostrar cómo un autor-traductor expresa sus sentimientos románticos y de libertad a través de la traducción.

Presentaremos a continuación en primer lugar el contexto histórico de esta traducción y el estatus de la traducción en Cuba en el siglo XIX. Luego hablaremos del traductor y del autor, Heredia y Legouvé, y más adelante analizaremos los dos textos, el original francés y su traducción en español, seguido de las conclusiones.

\section{Contexto histórico}

No se conoce la fecha exacta de la traducción pero síla de su primera publicación en 1821, en la Habana, en la revista fundada por el mismo traductor, Biblioteca de Damas. Luego se publicó en México en 1825 y más tarde en Nueva York en el mismo año. En 1832 fue reimpreso el poema con algunas modificaciones que Heredia le había hecho a su versión anterior. Esta nueva versión es considerada como su versión final y es la que será objeto del presente estudio. 
La traducción del poema de Legouvé se sitúa en el siglo XIX cubano, cuando Cuba era aun colonia española. A finales del siglo XVIII, los criollos comenzaban a tomar conciencia de la necesidad de un cambio en el régimen político y administrativo, es decir, la necesidad de poner fin a su estatus de colonia española. Era el momento de la búsqueda de la identidad nacional propia y la negación de una impuesta por los españoles que ya ciertamente no correspondía con la realidad de la época. Las opiniones eran diversas. Los cubanos estaban de acuerdo sobre lo que no querían, continuar bajo un régimen colonial, pero diferían en lo que deseaban en cuanto a sistema político y administrativo. ¿Acaso la mejor solución era el reformismo, el autonomismo, el anexionismo, el independentismo o el separatismo?

Papel importante desempeñó la literatura cubana de finales del siglo XVIII y de principios del XIX, en el despertar de la conciencia de libertad en el país, además de aportar una mejoría intelectual y espiritual a los cubanos. Esta literatura de la primera mitad del siglo XIX, que es la etapa que nos ocupa, era el fruto de escritores autodidactas con talento natural. La calidad y los temas de esta literatura fueron evolucionando paralelamente al sentimiento de libertad. La actividad literaria del país comenzaba a desarrollarse, se encontraban trabajos estéticamente pobres pero ricos en contenido nacional y de identidad. Como bien sabemos, pues lo vemos aún en nuestros días, el arte siempre ha servido de arma y de medio de expresión de los pueblos. ¿Quién mejor que los escritores para expresar los sentimientos de los cubanos? Estos escritores a través de sus obras perseguían que los cubanos tomaran conciencia de su destino histórico. Por tanto, durante estos años, predominaban la literatura patriótica, la literatura popular, los escritos filosóficos y periodísticos, y finalmente las traducciones, las cuales constituyeron por consiguiente un medio de expresión de la época.

\section{La traducción en Cuba en el siglo XIX}

Los traductores cubanos en el siglo XIX eran fundamentalmente escritores, fenómeno universal que se manifiesta igualmente en Hispanoamérica con ilustres personajes como Jorge Luis Borges, Andrés Bello, Octavio Paz, entre otros. Estos escritores evidentemente no tenían formación de traductores. Habían vivido en el extranjero, estuvieron exiliados, estudiaron idiomas y conocían otras culturas y literaturas. Sus traducciones eran mayormente imitaciones pues se servían de los textos extranjeros, principalmente de autores europeos y americanos, para transmitir un sentimiento propio de nacionalidad y de libertad. La actividad de traducción era característica de casi todos los escritores de la época. Su objetivo no era traducir, como traductor profesional, sino más bien servirse de estas obras extranjeras para cumplir con sus propósitos personales. Escritores como Domingo del Monte, José Fornaris, Rafael María de Mendive, Juan Clemente Zenea, José Agustín Quintero, los hermanos Sellen, Diego Vicente Tejera, Julián del Casal y José María Heredia y Heredia fueron los motores de la literatura cubana de esta primera mitad del siglo XIX, a través de sus escritos literarios, patrióticos y sus traducciones.

\section{José María Heredia y Heredia, traductor y Ernest Wilfrid Legouvé}

José María Heredia y Heredia (1803-1839) fue el primero de los grandes poetas líricos cubanos del siglo XIX. Fue el precursor de la literatura romántica en América y cuenta entre los escritores más afamados de América Latina. Heredia tuvo una vida corta, murió con 35 años, pero su vida fue plena y rica en escritos literarios. A la edad de seis años leía el francés corrientemente y ya había traducido las “Fables de Florián”. Vivió en Venezuela, en Cuba y en México. Las imágenes del mar, los paisajes tropicales, la libertad americana, la actualidad histórica y sus reflexiones sobre la naturaleza y la sociedad, sus fracasos amorosos, el amor platónico o frustrado, marcaron la vida y el espíritu de este autor, y por lo tanto su obra. Toda la obra de Heredia muestra los sentimientos y las experiencias de su vida, en Cuba o en el exilio. Toda su obra es una tragedia que refleja una realidad romántica como su vida misma. Tradujo a clásicos como Voltaire pero también autores de menos importancia. Retomaba los héroes de la antigüedad para expresar el mismo sentimiento de libertad, de opresión, de tiranía, pero en una época moderna. 
La poesía de Heredia tuvo también carácter patriótico. En 1822 ingresa en una organización revolucionaria, la logia Caballeros Racionales, una de las ramas del movimiento Soles y Rayos de Bolívar, y un año más tarde, una vez descubierta la conspiración, se ve forzado a exiliarse en los Estados Unidos para escapar así de la prisión.

Por su parte, Ernest Wilfrid Legouvé nació en 1807 y murió en 1903. Era un hombre de letras, poeta, autor drámatico y ensayista. Es reconocido por sus contribuciones al desarrollo y a la educación de la mujer en Francia, discursos sobre el tema que quedaron registrados en un volumen editado en 1888. En el poema en estudio, Legouvé considera a la mujer en su papel de esposa, hermana y madre; la mujer dejaba de ser un objeto de lujo, le daba un merecido valor. Es evidente que estas ideas en el siglo XIX eran muy revolucionarias.

\section{Análisis comparativo del poema "Le mérite des femmes" y de su imitación "El mérito de las mujeres".}

El objetivo del estudio de ambos poemas no es hacer un exhaustivo análisis literario, sino un análisis comparativo de dos obras desde el punto de vista de la actividad de traducción para determinar las estrategias de traducción del escritor cubano y así poner en evidencia sus intervenciones deliberadas.

Heredia no eligió el poema al azar. Heredia amó mucho a las mujeres en su corta vida. Desde la edad de 14 años cuando fue por segunda vez a Cuba después de haber vivido en México comenzó a amar. Pudiéramos mencionar entre los grandes amores de su vida: Lesbia o Belisa ${ }^{2}$, a quien dedicó poemas tales como "A mi querida" (1819), "La partida" (1819) o "El rizo del pelo" (1819) pero también escribió poemas de desamor como "La inconstancia” (1821) o "Misantropía" (1821); Lola ${ }^{3}$; Emilia o Pepilla ${ }^{4}$ y Jacoba Yañez, una mexicana con quien se casó en 1827. Pero para él, el lugar más importante estaba reservado a su madre, a quien escribía y amaba enormemente, como se puede ver en las múltiples cartas que él le escribía (Augier:2003).

Lo primero que salta a la vista es el título y notaremos que Heredia hizo una simple traducción literal del título original, cosa que no sorprende teniendo en cuenta que ese título no presenta ningún tipo de ambiguëdad ni se presta a diversas interpretaciones. El poema bien merece ese título pues es un homenaje a la mujer en todas las etapas de la vida.

En cuanto a su estructura, el poema original consta de 9 estrofas mientras que la traducción de Heredia tiene 19 pues el traductor agregó 10 estrofas. En cuanto a la medida de los versos de cada estrofa, tanto el poema original como la traducción poseen mayormente versos endecasílabos (11 sílabas) o dodecasílabos (12 sílabas), aunque en la traducción de Heredia podemos encontrar también versos amétricos o irregulares (versos con medida diferente en una misma estrofa). En cuanto a la rima de los versos, en el original, la rima es consonante (a partir de la vocal prosódicamente acentuada, incluida ella, coinciden todos los sonidos, vocálicos y consonánticos). Heredia cambió la rima de los versos en su traducción mezclando la rima libre con la rima consonante, un estilo característico de gran parte de su obra poética.

En cuanto a la primera estrofa, Heredia la eliminó y la sustituyó por una nueva. En el original, el autor habla de la modestia, de los grandes escritores; Heredia prefirió dedicar esta estrofa a Emilia, el amor de su vida y quien aparece en gran parte de su obra literaria, a manera de introducción del poema. En los siguientes versos observamos la intención del autor: ... Emilia bella, / honor y gloria de tu sexo hermoso, / admite con agrado el homenajel de mi fina amistad, y sé mi Musa... (línea 2 a la 5).

En la segunda estrofa Legouvé utiliza la palabra Beauté para calificar a la mujer. Heredia prefiere la palabra "Beldad" que en español encierra una mayor carga semántica que el equivalente más frecuente "Belleza". Su elección se justifica por el hecho de haber dedicado el poema a Emilia; Heredia utiliza la palabra "Beldad" para reafirmar su belleza.

En la tercera estrofa del original, Heredia elimina 9 versos, desde la línea 33 hasta la 42, a partir de...ses accords ont cessé, son maître la remplace... hasta... un bal suit le concert; c'est une autre merveille..., y construye además 2 estrofas a partir de esta tercera estrofa original. En la traducción (líneas 63 a la 98), Heredia agregó versos a partir de...! Oh bellas artes! hasta ... con él al seno suspendido..., con la intención de señalar el papel de la madre en la vida. Los versos 
agregados describen los cuidados de una madre, y la muestra de este amor es la introducción de la expresión "madre mía".

En la estrofa cuarta del original, línea 74, Legouvé utiliza la frase: ...il poursuit, il appelle un bonheur qu'il ignore:/ de qui le tiendra-t-il? C'est d'une femme encore... Con esta frase el autor describe toda la felicidad, la ebriedad que le brinda su primer amor. Para él, ... il nage dans un air tout chargé de bonheur... y ...et son heureuse vie est une longue extase... (líneas 89 y 111 respectivamente). Sin embargo, Heredia va a manipular esta frase para darle un nuevo sentido, pues su primer amor fue infeliz: ... no puede sacudir: anhela ardientel una felicidad desconocida, / y le perturba luego de repentel misterioso terror: su alma encendidal no puede hallar descanso... (línea 121 a la 125). Heredia quiere señalar de esta forma su sufrimiento anterior por su primer amor. Luego encuentra a Emilia,... pero te vi, adorada,/ y pensé ver a un dios... (líneas 127 y 128). A partir de la línea 157 Heredia agregó otra estrofa, es decir la sexta de la traducción, que no aparece en el original.

En la quinta estrofa del original, octava de la traducción, Legouvé habla del artesano (artesain) y del ministro (ministre), para expresar que todo hombre, poco importa la clase social, siente la necesidad al final de la jornada de trabajo, de encontrarse con su esposa para así olvidar el cansancio. Heredia mantiene el mismo sentido pero remplaza artesano por campesino,... del grave arado con la reja dura/ despedazando el rústico la tierra,/ sobre los surcos del sudor prodiga.... (Líneas 188, 189 y 190). Heredia brinda al texto un matiz más cubano al hablar del campesino, de la tierra, del arado, pues Cuba es un país agrícola. Con este cambio adapta el poema al contexto geográfico cubano.

En la sexta estrofa de la traducción, Heredia hace referencia a lo que él sentía durante el viaje en barco cuando tuvo que abandonar a su amada para exiliarse: ... del mar bañaba con furiosa espuma... (línea 158) o... el insondable espacio que tendido/ me apartaba de tí... (líneas 160 y 161) y luego dice... con placer melancólico, inefable,/ tu beldad recordaba... (líneas 167 y 168). Por otra parte, en la sexta estrofa del original, las líneas 190 hasta la 247 fueron eliminadas por Heredia: ...ce jeune homme rampait dans un repos vulgaire... hasta...leur bras comme leurs yeux leur donnait la victoire...

Heredia agrego allí un párrafo también dedicado a Emilia e introduce a Cuba, ubicando definitivamente el poema en la isla. Cuando dice Cuando fatal persecución en Cuba...(línea 359) hace referencia a la conspiración de Rayos y Soles de Bolívar, organización revolucionaria a la que Heredia pertenecía, que fue descubierta en 1823 por lo cual él tuvo que exiliarse en los Estados Unidos, de donde luego viajó a Mexico.

Estos son algunos de los ejemplos del análisis comparativo del poema original y de la traducción. Se observa que a todo lo largo de su traducción, Heredia agrega y elimina versos. Las tres últimas estrofas del original son un claro ejemplo más de este tipo de intervención del traductor.

\section{Conclusiones}

Con esta rápida comparación de la traducción de Heredia y el original en francés de Legouvé, se demuestra que el texto de partida fue objeto de una intervención deliberada por parte del escritor cubano. Por intervención deliberada del traductor se entiende: una estrategia que resulta de una libre elección del traductor, una estrategia visible, responsable, libre y arriesgada, una estrategia de traducción selectiva que consiste en resaltar o conservar sólo aquello que le interesa al traductor ${ }^{5}$.

El análisis comparativo de estas dos obras muestra que Heredia realizó intervenciones deliberadas acomodando por decisión propia el texto a sus necesidades e intereses; el traductor adapta, asimila y manipula como resultado de una elección personal libre.

Su público son sus lectores, pero también Émilie, quien escuchará una vez más el amor que él siente por ella. Conviene preguntarse hasta qué punto su público no es también él mismo. Heredia escribe para él, porque él es un romántico, porque ama a esta mujer, porque quiere servirse de este texto para expresar sus ideas. De allí que el original no es más que pretexto o materia prima para un nuevo poema. 
Como hemos observado, Heredia sigue un propósito personal (dedica su poema a Émilie), exalta el patriotismo cubano (habla del campesino y no del artesano, de la tierra y del arado; introduce el concepto de exilio y menciona Cuba; su poema, su amor, no tiene lugar en Francia, sino en la isla) y utiliza varias estrategias (elimina y agrega versos; cambia la estructura del poema original).

Con relación a su forma de traducir, Heredia escribió en su artículo "Poetas franceses modernos: Jacobo Delille" ${ }^{\text {: }}$

"El traductor que copia servilmente formas extranjeras, disfraza su idioma y su original, a quien no traduce, sino calumnia. El pintor que quiere hacer un retrato parecido toma la fisonomía; el que pretende expresar fielmente a un clásico, y conservar todos sus pensamientos, procure escribir como él habría escrito en aquella lengua, porque no deben traducirse las palabras, sino el genio".

La traducción ocupa un lugar significativo en toda su obra literaria. La actividad de traducción de Heredia consistió como hemos visto en intervenciones deliberadas a través de las cuales se apropiaba, imitaba la obra original. Sus traducciones son poesías inspiradas de un original, de ahí la denominación de poesía intervencionista que proponemos para sus traducciones. Definiremos poesía intervencionista como la manipulación de una obra original en el acto de traducir, por la cual el traductor-autor crea una obra completamente nueva: no traducir, no copiar, simplemente RECREAR.

En la obra literaria de José María Heredia, encontramos eso que Martí llamó "lo herédico".

“...esa alma que se consume, ese movimiento a la vez arrebatado y armonioso, ese lenguaje que centellea como la bóveda celeste, ese período que se desata como una capa de batalla y se pliega como un manto real, eso es lo herédico". (Retamar 1972:178-179)

es decir, lo romántico.

Heredia ocupa un lugar importante en la literatura cubana del siglo XIX. En toda su obra, encontramos el romanticismo excesivo resultante de una vida turbulenta, loca y peligrosa como un huracán, como él mismo la calificaba. Su poesía intervencionista, está llena de intervención deliberada. En un futuro trabajo veremos sin embargo que no todas las traducciones de Heredia fueron imitaciones. En efecto él diferenciaba cuándo iba a traducir y cuándo a imitar. Nos interesa examinar las razones precisas que lo llevaban a una u otra estrategia. Lo cierto es que el resultado de su proceso de traducción fue de una riqueza a la altura de su creación literaria.

\section{NOTAS}

1. Legouvé, Gabriel Jean Baptiste Ernest Wilfrid: (1807-1903), fue un hombre de letras, poeta, autor dramático y ensayista, célebre en el siglo XIX. Hijo del poeta y académico Gabriel-Marie Legouvé (1764-1812). Junto a su carrera de autor, Legouvé era un militante activo pour los derechos de las mujeres y la educación de los niños. Escribió L'Histoire morale des femmes (1848).

2. Dos apodos para la misma mujer de nombre Isabel Rueda (de quien no poseemos más informaciones).

3. Dolores Junco y Morejón, nombrada también por Heredia, Ninfa del San Juan o la Ninfa del Yumuri.

4. Josefa de Arango y Manzano, hija de José Arango y Castillo, amigo que le dio refugio y que lo ayudó a partir a los Estados Unidos luego de descubrirse la conspiración de Rayos y Soles de Bolívar.

5. Estas definiciones surgieron durante el Seminario Théories de la traduction en el semestre de otoño 2004 en la Universidad de Montreal, con el prof. Georges L. Bastin.

6. Miscelánea, Tlalpam, t. II, No 5, enero 1830. 


\section{BIBLIOGRAFÍA CONSULTADA}

Augier, Á. (2003): Obra poética de José María Heredia, La Habana, Letras cubanas.

BUENO, S. (1980): Figuras cubanas del siglo XIX, La Habana, Unión.

Claretie, L. (1904) : «Ernest Legouvé, souvenirs personnels et documents inédits », La Revue de Paris, mars-

avril 1904

Curbelo, J. D.: “ Para una historia de la traducción en Cuba ”, HISTAL,

<http://www.histal.umontreal.ca/espanol/documentos/para\%20una\%20historia\%20de\%20la\%20traduccion\%20en\%20

Cuba.htm>.

SuÁREZ LEÓN, C. : "Traducir y transgredir: Heredia como modelador de la cultura cubana”, HISTAL,

$<$ http://www.histal.umontreal.ca/espanol/documentos/traducir\%20y\%20transgredir\%20heredia\%20como\%20modelad or\%20de\%201a\%20cultura\%20cubana.htm $>$.

\section{ANEXO}

Estrofas escogidas para hacer el análisis comparativo del poema original y su traducción.

\begin{tabular}{|c|c|c|c|}
\hline \multicolumn{2}{|c|}{$\begin{array}{l}\text { Le mérite des femmes } \\
9 \text { estrofas }\end{array}$} & \multicolumn{2}{|c|}{$\begin{array}{l}\text { EI mérito de las mujeres } \\
19 \text { estrofas }\end{array}$} \\
\hline $\begin{array}{l}1 \\
2 \\
3 \\
4 \\
5 \\
6 \\
7 \\
8\end{array}$ & $\begin{array}{l}\text { Estrofa } 1 \\
\text { Juvénal, dans ses vers digne émule d'Horace, } \\
\text { Des préaux, qui tous deux les rendit au Parnasse, } \\
\text { Coutre un sexe, paré de vertus et d'attraits, } \\
\text { Du carquois satirique ont épuisé les traits. } \\
\text { De ces grands écrivains je marche loin encore; } \\
\text { Mais j'ose, défenseur d'un sexe que j'honore, } \\
\text { Opposant son pouvoir à leur inimitié, } \\
\text { Célébrer des humains la plus belle moitié. }\end{array}$ & $\begin{array}{l}1 \\
2 \\
3 \\
4 \\
5 \\
6 \\
7 \\
8 \\
9 \\
\end{array}$ & $\begin{array}{l}\text { Estrofa } 1 \\
\text { Canto las dulces gracias y virtudes } \\
\text { Que ornan a la mujer. Emilia bella, } \\
\text { Honor y gloria de tu sexo hermoso, } \\
\text { Admite con agrado el homenaje } \\
\text { De mi fina amistad, y sé mi Musa. } \\
\text { Yo lograré feliz la única gloria, } \\
\text { El solo premio a que en mi canto aspiro, } \\
\text { Si tierna me consagras un suspiro, } \\
\text { Y un lugar de cariño en tu memoria. }\end{array}$ \\
\hline $\begin{array}{l}13 \\
14 \\
15 \\
16\end{array}$ & $\begin{array}{l}\text { Fragmento de estrofa } 2 \\
\text { Et dans l'homme, enfanté par un plus grand miracle, } \\
\text { Eut fait le spectateur de ce nouveau spectacle, } \\
\text { Pour son dernier ouvrage il créa la Beauté. } \\
\text { On sent qu'à ce chef-d'œuvre il doit s'être arrêté. }\end{array}$ & $\begin{array}{l}19 \\
20 \\
21 \\
22 \\
\end{array}$ & $\begin{array}{l}\text { Fragmento de estrofa } 2 \\
\text { Y al hombre creó, del orbe soberano. } \\
\text { En la dulce Beldad, su obra postrera, } \\
\text { Se detuvo el Creador : : noble destino, } \\
\text { Que abrió a su gloria de feliz carrera! }\end{array}$ \\
\hline \multirow[t]{2}{*}{$\begin{array}{l}31 \\
32 \\
33 \\
34 \\
35 \\
36 \\
37 \\
38 \\
39 \\
40 \\
41 \\
42\end{array}$} & $\begin{array}{l}\text { Estrofa } 3 \\
\text { (...) L'œil tantôt sur Chloris, tantôt sur l'instrument, } \\
\text { Chacun goûte à longs traits ce double enchantement. } \\
\text { Ses accords ont cessé, son maître la remplace : } \\
\text { Il a plus de science, a-t-il autant de grâce? } \\
\text { Il enfante des sous plus pressés, plus hardis ; } \\
\text { Mais offre-t-il ces bras par l'amour arrondis, } \\
\text { Qui, s'étendant autour de la harpe savante, } \\
\text { L'enlacent mollement de leur chaîne vivante? } \\
\text { Offre-t-il la rougeur, le touchant embarras } \\
\text { Qui d'un front virginal augmentent les appas } \\
\text { Plaît-il enfin à l'œeil comme il séduit l'oreille? } \\
\text { Un bal suit le concert; c'est une autre merveille (...) }\end{array}$ & $\begin{array}{l}45 \\
46 \\
47 \\
48\end{array}$ & $\begin{array}{l}\text { Estrofa } 3 \\
\text { ¿Los pintaré? Del clave a los acentos } \\
\text { Cloris une su voz fácil y dulce, } \\
\text { (A partir de aquí Heredia elimina los versos } \\
\text { 33-42 del original y luego escribe) } \\
\text { Y yo la escucho estático y pasmado. } \\
\text { Su canto hermoso me penetra el alma, } \\
\text { Me enajena feliz, y arrebatado } \\
\text { En sublime placer, tiemblo y la adoro. }\end{array}$ \\
\hline & $\begin{array}{l}\text { Fragmento agregado por Heredia en su } \\
\text { traducción a la estrofa } 3 \text { del original }\end{array}$ & $\begin{array}{l}72 \\
73 \\
74 \\
\end{array}$ & $\begin{array}{l}\text { Graves censores } \\
\text { De la mujer, negad sus beneficios } \\
\text { Ella carga en el seno doloroso. }\end{array}$ \\
\hline
\end{tabular}




\begin{tabular}{|c|c|c|c|}
\hline & & $\begin{array}{l}82 \\
83 \\
84 \\
85 \\
86 \\
87 \\
88 \\
89 \\
90 \\
91 \\
92 \\
93 \\
94 \\
95 \\
96 \\
97 \\
98 \\
\end{array}$ & $\begin{array}{l}\text { (...) } \\
\text { ¡Oh maternal amor! Si el niño duerme, } \\
\text { Con vigilante oído } \\
\text { De las tinieblas al silencio atiende. } \\
\text { O si Morfeo la adormece un punto, } \\
\text { Al más leve rumor abre de nuevo } \\
\text { Los agravados párpados, y pronta, } \\
\text { A la cuna del hijo ansiosa vuela; } \\
\text { Por largo rato le contempla inmóvil, } \\
\text { La paz disfruta de su blando sueño, } \\
\text { Y a su lecho se vuelve, aun no tranquila. } \\
\text { Más si despierta el niño, } \\
\text { Le brinda grata en el ebúrneo seno } \\
\text { Vida, fuerza y salud en leche pura. } \\
\text { ¿Qué importa la fatiga a su ternura? } \\
\text { En su hijo existe, y al esposo amante } \\
\text { Se muestra más bella } \\
\text { Con él al seno suspendido. }\end{array}$ \\
\hline & Fragmento de estrofa 4 & & Fragmento de estrofa 5 \\
\hline 74 & Il poursuit, il appelle un bonheur qu'il ignore : & 120 & Y el insufrible peso que le oprime \\
\hline 75 & De qui le tiendra t-il? c'est d'une femme encore. & 121 & No puede sacudid: anhela ardiente \\
\hline 76 & Une femme, en secret lui rendant ses soupirs, & 122 & Una felicidad desconocida, \\
\hline 77 & Rêveuse, s'abandonne à ses vagues désirs. & 123 & Y le perturba luego de repente \\
\hline 78 & O première faveur d'une première amante! & 124 & Misterioso terror: su alma encendida \\
\hline 79 & Dès que, sur l'incarnat d'une bouche charmante, & 125 & No puede hallar descanso ... \\
\hline 80 & Il a bu des baisers le nectar inconnu, & 126 & De este modo \\
\hline 81 & Dès qu'un autre succès, par degrés obtenu, & 127 & Sufrí también; pero te ví, adorada, \\
\hline 82 & Va conduit dans les bras de sa belle maîtresse & 128 & Y pensé ver a un dios. Estremecido, \\
\hline 83 & De surprise en surprise, au comble de l'ivresse, & 129 & Con débil planta, respirando apenas, \\
\hline 84 & Comme un aveugle, à qui l'art rendrait la clarté, & 130 & Y en confusión dulcísima perdido \\
\hline 85 & Dans un autre univers il se croit transporté ; & 131 & Me sentí a tu mirar...jHoras felices! \\
\hline 86 & Il ne se connaît plus, il palpite, il soupire ; & 132 & ¡Oh languidez sublime y deliciosa! \\
\hline 87 & Il se sent étonné du charme qu'il respire ; & 133 & ¡Oh, cuánto fui feliz! ¡Cuánto, mi hermosa, \\
\hline 88 & L'ivresse de ses sens a passé dans son cœur ; & 134 & Mi sangre ardió, cuando a tus labios puros \\
\hline 89 & Il nage dans un air tout chargé de bonheur. & 135 & El beso arrebate...! Cual desgraciado \\
\hline 90 & Sa maîtresse! oh! combien sou regard la dévore! & 136 & En tinieblas nacido, a quien el arte \\
\hline 91 & Il la voit comme un dieu que sans cesse il adore ; & 137 & Hiciera ver la luz, arrebatado \\
\hline 92 & Son cœur brûlait hier, son cœur brûle aujourd'hui ; & 138 & A otro universo entonces me creyera: \\
\hline 93 & Il ne sait s'il existe ou dans elle on dans lui. & 139 & Hablar contigo, verte y adorarte \\
\hline 94 & Paraissent-ils ensemble au milieu d'une fête ? & 140 & Mi ocupación y mi delicia fuera. \\
\hline \multirow[t]{2}{*}{95} & Son oeil préoccupé ne suit que sa conquête. & 141 & Tú encantaste mis horas: la carrera \\
\hline & $(\ldots)$ & 142 & De mi vida feliz ornaste en flores: \\
\hline 102 & Le rayon du matin, c'est la douce lumière & 143 & Por tí la paz, la risa y los amores \\
\hline 103 & Qui luit si tendrement sous leur longue paupière ; & 144 & En torno de mi frente revolaban, \\
\hline 104 & Le murmure flatteur des limpides ruisseaux, & 145 & Y gratos alejaban \\
\hline 105 & Le souffle des zéphyrs, le concert des oiseaux, & 146 & Los cuidados, angustias y dolores. \\
\hline 106 & C'est le son de la voix qui répond à son âme ; & 147 & ¡Oh! ¡cuánto padecí cuando arrancado! \\
\hline 107 & Tout l'univers enfin l'entretient de sa flamme. & 148 & Me vi a tu dulce amor y a tu presencia! \\
\hline \multirow[t]{2}{*}{108} & Pour lui plus de langueurs, plus de maux, plus d'ennuis ; & 149 & Dilo tú, joh noche! que testigo fuiste \\
\hline & L'amour remplit, enchante et ses jours et ses nuits & 150 & De mi acerbo penar, de mis furores. \\
\hline 109 & Il n'a qu'un seul objet qui l'occupe et l'embrase & 151 & Cuenta como mi llanto recibías, \\
\hline 110 & Et son heureuse vie est une longue extase. & 152 & Compasiva mis quejas escuchabas, \\
\hline 111 & & 153 & Y en tu grato silencio mitigabas \\
\hline
\end{tabular}




\begin{tabular}{|c|c|c|c|}
\hline & & 154 & El tormentoso horror de aquellos días. \\
\hline & $\begin{array}{l}\text { Estrofa 6, agregada por Heredia en su } \\
\text { traducción que sigue a la estrofa } 4 \text { del original }\end{array}$ & $\begin{array}{l}157 \\
158 \\
159 \\
160 \\
161 \\
164 \\
165 \\
166\end{array}$ & $\begin{array}{l}\text { (...) Mas no a mi corazón; sobre alta roca } \\
\text { Del mar bañaba con furiosa espuma, } \\
\text { Salvaba mi agitada fantasía } \\
\text { El insondable espacio que tendido } \\
\text { Me apartaba de ti: mi pecho ardía... } \\
\text { (...) Canté los males de la ausencia fiera } \\
\text { Al echo incierto, al áspero silbido } \\
\text { Del viento bramador... }\end{array}$ \\
\hline $\begin{array}{l}131 \\
132 \\
133 \\
134 \\
135 \\
136 \\
137 \\
138 \\
139\end{array}$ & $\begin{array}{l}\text { Fragmento de la estrofa } 5 \\
\text { Dès l'aube, en longs travaux l'artisan se prodigue : } \\
\text { Sous le fardeau, le soir, il succombe affaissé ; } \\
\text { Il revoit sa compagne, et se sent délassé. } \\
\text { Le ministre languit dans son pouvoir suprême : } \\
\text { Au sein de sa compagne il vient se fuir lui-même. } \\
\text { Il y vient oublier l'ennui, le noir soupçon, } \\
\text { Dont à l'âme des grands s'attache le poison ; } \\
\text { Et, distrait de l'orgueil par l'amour qui l'appelle, } \\
\text { Du poids, de ses honneurs il respire auprès d'elle. }\end{array}$ & $\begin{array}{l}186 \\
187 \\
188 \\
189 \\
190 \\
194 \\
195 \\
200 \\
201\end{array}$ & $\begin{array}{l}\text { Fragmento de la estrofa } 8 \\
\text { De una esposa el afecto, la dulzura, } \\
\text { Doquier del hombre templan la fatiga. } \\
\text { Del grave arado con la reja dura } \\
\text { Despedazando el rústico la tierra, } \\
\text { Sobre los surcos del sudor prodiga. } \\
\text { (...) } \\
\text { El ministro imperioso } \\
\text { Que a reinos manda con altiva frente } \\
\text { (...) } \\
\text { Por amor del orgullo distraído, } \\
\text { Respira a par de su sencilla esposa (...) }\end{array}$ \\
\hline & Esta estrofa la agregó Heredia en su traducción & $\begin{array}{l}155 \\
156 \\
157 \\
158 \\
159 \\
160 \\
161\end{array}$ & $\begin{array}{l}\text { Estrofa } 6 \\
\text { Levantábase el sol, y al universo } \\
\text { La claridad tornaba y alegría, } \\
\text { Más no a mi corazón sobre alta roca } \\
\text { Del mar bañaba con furiosa espuma, } \\
\text { Salvaba mi agitada fantasía } \\
\text { El insondable espacio que tendido } \\
\text { Me apartaba de ti: (...) }\end{array}$ \\
\hline $\begin{array}{l}367 \\
368 \\
369 \\
370 \\
371\end{array}$ & $\begin{array}{l}\text { Estrofa } 17 \text { agregada por Heredia en su } \\
\text { traducción a la estrofa } 9 \text { del original } \\
\text { Fragmento de la estrofa } 9 \\
(\ldots) \\
\text { Toutes enfin, l'appui des Français malheureux, } \\
\text { Parlaient, priaient, pleuraient, ou s'immolaient pour eux, } \\
\text { Leur âme en nos dangers fut toujours secourable. } \\
\text { Remontons au moment où d'un règne exécrable } \\
\text { Septembre ouvrit le long et vaste assassinat. } \\
(\ldots)\end{array}$ & $\begin{array}{l}359 \\
360 \\
361 \\
362 \\
363 \\
364 \\
365 \\
366 \\
367 \\
368 \\
369 \\
370 \\
371 \\
372 \\
373 \\
\end{array}$ & $\begin{array}{l}\text { Cuando fatal persecución en Cuba } \\
\text { Turbó la dulce paz con sus furores. } \\
\text { ¿Olvidarte podré, celeste Emilia, } \\
\text { Que habitabas el techo hospitalario } \\
\text { Donde a la proscripción enfurecida } \\
\text { Oculté, a mi pesar, mi amarga vida? } \\
\text { ¡Oh! ¡cómo la piedad, hija del cielo, } \\
\text { En tu divina frente disipaba } \\
\text { De tu amigo proscripto los dolores! } \\
\text { ¡Ángel de dulce paz y de consuelo! } \\
\text { Tu plácida memoria, que embellece } \\
\text { De mi destierro las cansadas horas, } \\
\text { Hasta el sepulcro bajará conmigo, } \\
\text { Y en su yelo no mas podrá entibiarse } \\
\text { La gratitud ardiente de tu amigo. }\end{array}$ \\
\hline
\end{tabular}

